\title{
Cerebrovascular events in COVID-19 patients
}

\author{
Ajay Kumar Mishra ${ }^{1}$, Kamal Kant Sahu ${ }^{1}$, Anu Anna George ${ }^{1}$, Jennifer Sargent ${ }^{1,2}$, Amos Lal $^{3}$ \\ ${ }^{1}$ Department of Internal Medicine, St. Vincent Hospital, Worcester, MA; ${ }^{2}$ University of Massachusetts Medical School, \\ Worcester, MA; ${ }^{3}$ Department of Internal Medicine, Division of Pulmonology and Critical Care Medicine, Mayo Clinic, \\ Rochester, MN, USA
}

\begin{abstract}
Neurological manifestations in patients with COVID-19 are more frequently being reported. Cerebrovascular events have been reported in around $3 \%$ of patients. In this review we summarize the published literature on cerebrovascular events in patients with COVID-19 as available on the PubMed database. So far, 3 studies have reported cerebrovascular events. Cerebrovascular events were identified on screening patients with decreased consciousness or in the presence of focal neurological deficits. These events were common in elderly, critically ill patients and in patients with prior cardio-cerebrovascular comorbidities. The diagnosis of cerebrovascular events was confirmed with computed tomography of the brain in most studies reporting neurological events. Multiple pathological mechanisms have been postulated regarding the process of neurological and vascular injury among which cytokine storm is shown to
\end{abstract}

Correspondence: Amos Lal, Division of Pulmonary and Critical Care Medicine, Mayo Clinic, Rochester, MN 55902, USA.

E-mail: Lal.Amos@mayo.edu - manavamos@gmail.com

https://orcid.org/0000-0002-0021-2033

Key words: COVID-19, cerebrovascular events, imaging, mechanism, outcome.

Conflict of Interest: The Authors have no conflict of interest to declare.

Ethical statement: The article does not contain the participation of any human being and animal.

Authors' contribution: All the authors made a substantive intellectual contribution. All the authors have read and approved the final version of the manuscript and agreed to be accountable for all aspects of the work.

Funding: None

Received for publication: 26 April 2020.

Accepted for publication: 4 June 2020.

${ }^{\circ}$ Copyright: the Author(s), 2020

Licensee PAGEPress, Italy

Monaldi Archives for Chest Disease 2020; 90:1341

doi: 10.4081/monaldi.2020.1341

This article is distributed under the terms of the Creative Commons Attribution Noncommercial License (by-nc 4.0) which permits any noncommercial use, distribution, and reproduction in any medium, provided the original author(s) and source are credited. correlate with mortality. Patients with severe illness are found to have a higher cardio- cerebrovascular comorbidity. With an increasing number of cases and future prospective studies, the exact mechanism by which these cerebrovascular events occur and attribute to the poor outcome will be better understood.

\section{Introduction}

After starting in December 2019 at Wuhan, China, the novel coronavirus 19 (COVID-19) infection has become a pandemic of global concern. As of $20^{\text {th }}$ April, more than 2.4 million cases have been reported in 185 countries [1]. A continually rising number of patients with COVID-19 continues to be a challenge to the medical community and is a major public health concern for experts of all medical fields. This severe acute respiratory syndrome coronavirus -2 is an RNA virus that predominantly presents with fever, fatigability, respiratory symptoms including nonproductive cough and shortness of breath. Multiple organ involvement including cardiac, gastrointestinal, reticuloendothelial system and neurological involvement have been reported [2-4]. Neurological manifestations are being reported infrequently. In a study by Mao et al., $36.4 \%(n=78)$ of patients had evidence of neurological involvement, among which $24.8 \%$ ( $n=53$ ) of patients had evidence of central nervous system (CNS) involvement. Among those who had CNS involvement, cerebrovascular events were reported in $2.8 \%$ $(n=6)$ patients [5].

\section{Methodology}

In this review we summarize the published literature on cerebrovascular event in patients with COVID-19 as available on the PubMed database. We have included original studies that have reported adult patients with cerebrovascular events as confirmed by neurological imaging. We have excluded studies including children, and articles under opinion and comments category. All the articles published before $20^{\text {th }}$ April 2020 have been included in this review.

\section{Clinical presentation}

Studies reporting neurological involvement have reported clinical features of headache, dizziness, impaired vision, decreased consciousness, agitation, presence of ataxia, and seizure in patients with COVID-19 [5-8]. However, the symptoms 
described have not been attributed to any specific etiological factor including encephalitis, vasculitis, and cerebrovascular event. In most scenarios patients have been evaluated for neurological involvement following the identification of neurological symptoms $[5,6]$. Interestingly two patients have been reported to present with hemiplegia and later diagnosed to have COVID-19 based on their computed tomography (CT) of lungs revealing typical findings. In the same study, the median duration to the onset of a cerebrovascular event following hospital admission was reported to be nine days. While details of CNS examination are mostly limited, confusion as assessed by confusion assessment method, exaggerated tendon reflects, clonus and extensor plantar reflex have been reported frequently $[5,9]$. However, most reported examination findings have been bilateral suggesting a diffuse CNS process. Clinical findings specific to patients with imaging evidence of cerebrovascular event have not been reported in studies so far. Unlike other infections no specific pattern of neurovascular involvement has been reported [10-12].

\section{Imaging}

Computed tomography (CT) brain and magnetic resonance imaging (MRI) of the brain has been performed in COVID-19 patients with CNS involvement. CT brain (non-contrast) has been done and reported more frequently, owing to the ease of availability. MRI brain has been reported in a lesser number of patients [5,9,12-14]. In one study reporting MRI brain findings showed perfusion abnormalities in all $11(100 \%)$ patients and revealed evidence of ischemic stroke in $3(23 \%)$ patients $[5,9,13]$. These studies did not report the site, laterality, number and pattern of ischemic events.

\section{Mechanism}

While the exact mechanism by which COVID-19 leads to multi-organ dysfunction and mortality is still being studied, several key factors contributing to the same have been identified.

i) It has been shown that COVID-19 similar to other coronaviruses use angiotensin-converting enzyme 2 (ACE-2) receptor for entering into the cells. ACE -2 receptor is noted to be expressed in the lungs, heart, kidneys, and endothelium. Recent literature has shown evidence of endothelial inflammation in multiple vascular beds. Autopsy findings have revealed the presence of viral elements and inflammatory cells within the endothelium. Whether ACE-2 receptor involvement is directly related to myocardial, cerebrovascular injury is still a matter of debate [15-17].

ii) The exaggerated, continuous, dysfunctional and uncontrolled activation of the immune system causing excessive cytokine release has been consistently reported in patients with severe pulmonary disease and cardiac involvement. Cytokine storm is diagnosed with the presence of elevated plasma markers of inflammation like C-reactive protein (CRP), erythrocyte sedimentation rate (ESR), procalcitonin, ferritin, interleukins (IL2, IL-7), granulocyte - colony- stimulating factor and tumor necrosis alpha. In the studies reporting CNS involvement and cerebrovascular events, a significant difference in the levels of these inflammatory markers has not been proven to predict clinical presentation or outcome $[1,2,15]$. iii) Embolic events as noted by elevated d-dimer, serum cardiac markers including high-sensitivity troponin and proBNP have been consistently reported. Patients are also reported to be at a higher risk of arrhythmia secondary to myocarditis possibly precipitating embolism $[5,15,16,18]$.

iv) Other postulated mechanisms have been a) A prothrombotic state as noted by elevated d-dimer, fibrinogen, lactate dehydrogenase and other markers of coagulation, b) hypoxia induced ischemia and apoptosis, c) secondary to hemophagocytic lymphohistiocytosis, d) possible disruption of plaque secondary to the presence of risk factor and inflammation, e) coagulopathy and thrombocytopenia $[6,9,19,20]$.

While multiple mechanisms have been postulated cytokine storm has been shown to correlate with the severity of illness, acute respiratory distress syndrome (ARDS), acute cardiac injury, acute renal failure, the requirement of invasive and noninvasive mechanical ventilation, morbidity (hospital and ICU length of stay) and mortality $[2,15,18,21]$.

\section{COVID-19 in patients with prior cerebrovascular disease}

Most studies have reported a history of cardiovascular and cerebrovascular disease (CVD) together in comorbidities. Most of the available literature also supports the presence of cardio-cerebrovascular comorbidities as a predictor for severe illness $[8,15]$. Guan et al. reported the presence of cerebrovascular disease to be an independent factor linked with poor outcome. They included 1099 patients among which $1.4 \%(n=15)$ had a history of cerebrovascular disease. In this study, they also showed that the presence of cerebrovascular disease was higher among patients with severe illness $(2.3 \%)$ as compared to patients with mild illness $(1.1 \%)$ [22]. However, this was not statistically significant. Risk factors of cerebrovascular disease including older age, hypertension, diabetes, hyperlipidemia, smoking is also significantly higher among patients with severe infection [15,18,23]. Aggarwal et al. did a pooled analysis of the published literature on severity of COVID-19 among patients with past history of CVD. They identified 6 studies of COVID-19 which included patients with prior CVD. They independently analyzed four studies comparing presence of cerebrovascular disease in severe vs non-severe COVID19 cases. Interestingly they reported that patients with history of CVD had a 2.5-fold increase in odds of severe COVID-19 illness [24]. All these studies have not been able to show an association of increased mortality with prior CVD [25].

\section{Outcome}

Mortality in patients with COVID-19 is $2-4 \%$ in general population, which is higher as compared to seasonal flu. Mortality in critically ill patients is as high as $49 \%$ [2]. Advanced age, ARDS, acute cardiac injury, prior vascular comorbidities, elevated inflammatory markers are linked with mortality [2,5]. Diffuse CNS involvement has been reported to be higher among patients with severe illness. Only three studies have specifically reported patients with newly diagnosed cerebrovascular disease as shown in Table 1 . The study by Mao et al. reported that the presence of cerebrovascular disease as identified by the CT brain (non-contrast) 
Table 1. Showing studies reporting cerebrovascular events among patients with COVID-19.

\begin{tabular}{lccc} 
& Mao et al. [5] & Helms et al. [9] & Klok et al. [16] \\
Study type & Retrospective & Retrospective & Prospective \\
Setting & In patient & ICU & ICU \\
\hline Patients & $53(24.8 \%)$ & 58 & 184 \\
CNS involvement & $6(2.8 \%)$ & $49(84 \%)$ & 3 \\
\hline CVD & CT (Non-contrast) & 3 & MRI \\
Type of imaging & Not specified & Not specified & CT (Non-contrast) \\
Type of stroke & + & - & Not specified \\
Predictor of severity &
\end{tabular}

was higher among patients with severe infection and it was statistically significant. The other two studies were conducted in patients that were directly admitted to ICU due to higher severity of illness at onset $[5,9,16]$. However, these two studies did not compare morbidity or mortality in the subset of patients developing new onset cerebrovascular disease (with rest of the patients) owing to low event rates.

\section{Future direction}

Most studies have failed to report the role of medications including agents affecting renin -angiotensin- aldosterone, statins, antiplatelet agents, and anticoagulants uniformly $[18,21,26]$. The roles of CNS depressants including opioids, benzodiazepines, neuromuscular blocking agents on case identification and screening for neurological deficits among critically ill patients still needs consideration [12,13,19]. Multiple medications that are used for treatment, their role in cerebrovascular events and long term outcome still require clarification [12,14,27]. The exact mechanisms with which patients suffer cerebrovascular events and how these events attribute to poor outcome require further understanding [28].

\section{References}

1. Sahu KK, Mishra AK, Lal A. Comprehensive update on current outbreak of novel coronavirus infection (2019-nCoV). Ann Transl Med 2020;8:393.

2. Wu Z, McGoogan JM. Characteristics of and important lessons from the coronavirus disease 2019 (COVID-19) outbreak in China: summary of a report of 72314 cases from the Chinese Center for Disease Control and Prevention. Jama 2020. doi: 10.1001/jama.2020.2648.

3. Sahu KK, Lal A, Mishra AK. Latest updates on COVID-2019: A changing paradigm shift. J Med Virol 2020. doi: 10.1002/jmv.25760.

4. Mishra AK, Sahu KK, Lal A, Sargent J. Patterns of heart injury in COVID-19 and relation to outcome. J Med Virol 2020. doi: 10.1002/jmv. 25847

5. Mao L, Jin H, Wang M, et al. Neurologic manifestations of hospitalized patients with coronavirus disease 2019 in Wuhan, China. JAMA Neurol 2020. doi: 10.1001/jamaneurol. 2020.1127.
6. Wu Y, Xu X, Chen Z, et al. Nervous system involvement after infection with COVID-19 and other coronaviruses. Brain Behav Immun 2020. doi: 10.1016/j.bbi.2020.03.031

7. Kuriakose CK, Mishra AK, Vanjare HA, et al. Visual disturbance in patients with cryptococcal meningitis: The road ahead. J Neurosci Rural Pract 2017;8:151-2.

8. Grasselli G, Zangrillo A, Zanella A, et al. Baseline characteristics and outcomes of 1591 patients infected with SARS-CoV-2 admitted to ICUs of the Lombardy Region, Italy. JAMA 2020;323:1574-81. doi: 10.1001/jama.2020.5394

9. Helms J, Kremer S, Merdji H, et al. Neurologic features in severe SARS-CoV-2 infection. N Engl J Med 2020;382:226870. doi: 10.1056/NEJMc2008597.

10. Mishra A, Binu A, Abraham G, et al. Cerebrovascular injury following scorpion sting and snake envenomation: a case series. Can J Neurol Sci 2018;45:669-74.

11. Vanjare H, Mannam P, Mishra A, et al. Brain imaging in cases with positive serology for dengue with neurologic symptoms: a clinicoradiologic correlation. Am J Neuroradiol 2018;39: 699-703.

12. Dafer RM, Osteraas ND, Biller J. Acute stroke care in the coronavirus disease 2019 pandemic. J Stroke Cerebrovasc Dis 2020. doi: 10.1016/j.jstrokecerebrovasdis.2020.104881

13. Mishra AK, Arvind VH, Muliyil D, et al. Cerebrovascular injury in cryptococcal meningitis. Int J Stroke 2018;13:57-65.

14. Jin H, Hong C, Chen S, et al. Consensus for prevention and management of coronavirus disease 2019 (COVID-19) for neurologists. Stroke Vasc Neurol 2020. doi: 10.1136/svn-2020000382.

15. Driggin E, Madhavan MV, Bikdeli B, et al. Cardiovascular considerations for patients, health care workers, and health systems during the coronavirus disease 2019 (COVID-19) pandemic. J Am Coll Cardiol 2020;75:2352-71. doi: 10.1016/ j.jacc.2020.03.031

16. Klok F, Kruip M, van der Meer N, et al. Incidence of thrombotic complications in critically ill ICU patients with COVID19. Thromb Res 2020;191:145-7.

17. Varga Z, Flammer AJ, Steiger P, et al. Endothelial cell infection and endotheliitis in COVID-19. Lancet 2020;395:1417-8. doi: 10.1016/S0140-6736(20)30937-5.

18. Clerkin KJ, Fried JA, Raikhelkar J, et al. Coronavirus disease 2019 (COVID-19) and cardiovascular disease. Circulation 2020;141:1648-55. doi: 10.1161/CIRCULATIONAHA.120. 046941 .

19. Mishra AK, Sahu KK, Sargent JB. Cardiac drugs and outcome in COVID-19. QJM 2020. doi: 10.1093/qjmed/hcaa127

20. Sahu KK, Lal A, Mishra AK. An update on CT chest findings 
in coronavirus disease-19 (COVID-19). Heart Lung 2020. doi: 10.1016/j.hrtlng.2020.03.007

21. Bernheim A, Mei X, Huang M, et al. Chest CT findings in coronavirus disease-19 (COVID-19): relationship to duration of infection. Radiology 2020;295:200463. doi: 10.1148/ radiol.2020200463.

22. Guan W-J, Ni Z-Y, Hu Y, et al. Clinical characteristics of coronavirus disease 2019 in China. Ne Engl J Med 2020;382:170820. doi: 10.1056/NEJMoa2002032.

23. Mishra AK, Lal A, George AA. Letter by Mishra et al Regarding Article, "Infection as a Stroke Trigger: Associations Between Different Organ System Infection Admissions and Stroke Subtypes". Stroke 2019;50:e328. doi: 10.1161/ STROKEAHA.119.026990.

24. Aggarwal G, Lippi G, Henry BM. Cerebrovascular disease is associated with an increased disease severity in patients with Coronavirus disease 2019 (COVID-19): A pooled analysis of published literature. Int J Stroke 2020;15:385-9. doi: 10.1177/1747493020921664.

25. Mishra AK, Lal A, Sahu KK, et al. Mechanisms of neurological injury in COVID-19. World Neurosurg 2020. doi:10.1016/j.wneu.2020.05.089.

26. Mishra AK, Sahu KK, Lal A. Reporting of all cardiac medications and their outcome in COVID-19. J Med Virol 2020. doi: 10.1002/jmv.25843.

27. Mishra AK, Aaron S, Abhilash K, et al. Simple telephone call a feasible, useful and acceptable method of following up patients with cerebrovascular accidents: Prospective Cohort study in South India. Int J Stroke 2016;11:NP87-8. doi: $10.1177 / 1747493016654486$

28. Vincent J-L, Taccone FS. Understanding pathways to death in patients with COVID-19. Lancet Resp Med 2020;8:430-2. doi: 10.1016/S2213-2600(20)30165-X. 